\title{
Best Practices in Practice
}

\section{Efficient Staffing Patterns}

The primary challenge for oncology practices is maintaining high-quality care in the face of limited reimbursement and other changes in the health care environment. Efficiency is key to success, and developing staffing patterns that capitalize on the expertise of various members of the health care team is a critical component of practice efficiency.

In the wake of the Medicare Prescription Drug

Improvement and Modernization Act (MMA) of 2003, it is especially important for oncology practices to closely monitor drug inventory. However, it is equally important to set priorities and carefully manage physicians' time and staffing patterns. The use of midlevel providers can help reduce responsibilities once borne by physicians, freeing them to see more patients. In addition, the specific use of other clinical and administrative staff can help streamline workflow. The result is high-quality care, increased patient satisfaction, lower costs, and increased revenue.

One example of an oncology practice with efficient staffing patterns is New Hampshire Oncology-Hematology Practice. This nine-physician practice was established in 1976 by A. Collier Smyth, MD, the first board-certified oncologist in community practice in New Hampshire. Terry Steiner, director of operations, says the practice has enjoyed "amazing growth" over the past few years, as the use of midlevel providers has enabled physicians to see more new patients. More importantly, she adds, "The quality of care is incredibly better."

When the practice started, there was one physician assistant who saw patients every day. "However, we found that we had no room to accommodate walk-in patients or sick patients," says Steiner. The practice now includes six midlevel providers (four physician assistants and two nurse practitioners) who work across the five practice sites. Ms. Steiner explains that one physician assistant sees patients all day, while another is a back-up to the physician during hospital rounds and then sees add-on patients at the office. A physician assistant also completes all preliminary paperwork for hospital admissions from the office, saving time for both the physician and the patient.

A pharmacist was brought on board about a year ago and "has been instrumental in helping the practice succeed in the face of Center for Medicare and Medicaid Services (CMS) changes," according to Steiner. The pharmacist manages drug inventory, ensures compliance with new pharmacy regulations, and provides in-service education on new drugs when they are introduced into the practice. Some of these tasks had once been the responsibility of the midlevel providers in the practice, and the addition of the pharmacist has allowed them to focus more time on patients.

For example, patients coming to a site for chemotherapy did not always see a provider in the past. Patients now see a physician or midlevel provider at every visit. "If the patient is sick, chemotherapy is not given and so drugs aren't wasted," explains Steiner. "Nurses are integrally involved with patients during chemotherapy visits. They talk to patients about their overall health and are attentive throughout the infusion, noting adverse events. The nurses can now be proactive rather than reactive."

Wilshire Oncology Medical Group, in Pomona, California, is another practice that has kept pace with efficient staffing patterns. "When medical oncology became a specialty, we introduced nurses into the practice to work alongside the physicians," says Wendy McNatt, practice management administrator, who has been with the practice since 1975. "In the 1990s, with cuts in reimbursement, we added skilled medical assistants to work with both our nurses and our physicians. We brought in nurse practitioners in 1996 because of case volume." The approach has enabled the practice to save money while maintaining a high level of care.

McNatt notes that careful evaluation of staffing needs is important. For example, when the workload recently indicated that another nurse should be added to the practice, she reviewed the actual needs and instead hired a medical assistant. The medical assistant works in the chemotherapy room, performing such tasks as monitoring vital signs, answering phones, and making appointments, enabling nurses to spend more time with patients during administration of chemotherapy. Nurses are able to perform more direct patient care functions as a result of another medical assistant who works with the physicians and a pharmacy technician who mixes chemotherapy drugs.

Other staff in the 11-physician practice include two nurse practitioners. These midlevel providers see patients alongside the physician in the morning and work independently in the afternoon on chemotherapy follow-up and chart rounds. McNatt also credits the practice's efficiency on part-time office staff recruited from nearby colleges, who prepare charts, scan documents into the 
electronic medical record, and carry out a variety of other administrative tasks. "These workers are invaluable in getting us ready for the next day," she says.

Commenting on other components of practice efficiency in the changing health care climate, McNatt notes the importance of maintaining costs: "We price out each regimen to make sure we're not 'upside down' on them. Also, the physicians in the practice work very hard at establishing treatment guidelines for every disease. We want to ensure high quality care, but we can't afford to lose money." She adds, "We always thought we knew what our costs were. But we didn't really understand. Now, every nickel is costed out, and we know exactly what our costs are." She says that she also analyzes health care plans and has discontinued contracts because of limited reimbursement policies.

At the center of it all for both practices is the patient. "Our biggest challenge is to continue to provide the care we've built our reputation on and to continue to provide care in the office. It is truly a challenge not to shift the patient to a hospital setting, where a two-hour chemotherapy infusion could mean an overnight stay," says McNatt. Adds Steiner, "Oncologists need to be reimbursed fairly for all the work they do in order to stay in business. The system was broken and needs to be fixed. It hasn't been fixed yet. Now we fear that other insurers will jump on the CMS bandwagon before we have a chance to get the system working in a way that allows us to continue providing the quality of care everyone is used to receiving in a private practice setting."

Physicians and practice administrators can learn more about the critical elements of success through ASCO's half-day Practice Management Curriculum. The curriculum consists of three modules: Adapting to Changes in Medicare, Generating Practice Efficiencies, and Organizing for Service
Expansion. ASCO State/Regional Affiliates may request that the curriculum be presented at their local meeting.

\section{Increasing Patient Flow*}

A key element of practice efficiency is to increase patient flow, or the number of patients seen by the physician each day. This increased patient flow leads to better patient care, greater patient satisfaction, and increased revenue.

To achieve a higher patient flow, physicians should

- communicate with referring physicians.

- see new patients.

- be seen at the hospital, participate in medical staff life.

- see follow-up patients on a regular, clinically appropriate basis.

- delegate some follow-up visits to other providers (such as physician assistants, nurse practitioners, or registered nurses), as appropriate.

- ensure the quality of care throughout practice.

Physicians should not

- routinely be late for clinic.

- spend time filling out forms (disability, tumor registry).

- provide routine patient education.

- return routine patient phone calls (prescription refills, etc.).

- micromanage staff.

- undermine authority of administrator.

Administrators should

- ensure that there are adequate exam rooms for each physician.

- provide appropriate patient scheduling individualized by physician, if necessary.

- use other staff (clinical and administrative) to free up physician time whenever possible.

*From ASCO Practice Management Curriculum available at http://www.asco.org/ac/1,1003,_12-002877,00.asp. 